\title{
émulations
}

\section{Juan José Bautista S. - ¿Qué significa pensar desde América Latina?: Hacia una racionalidad transmoderna y postcolonial}

\author{
Luis Martínez Andrade
}

Émulations - Revue de sciences sociales

2015, «Comptes rendus critiques, En ligne »

\section{Article disponible à l'adresse suivante}

https://ojs.uclouvain.be/index.php/emulations/article/view/7073

\section{Pour citer cet article}

Luis Martínez Andrade, « Juan José Bautista S. - ¿Qué significa pensar desde América Latina?: Hacia una racionalidad transmoderna y postcolonial », Émulations, en ligne. Mise en ligne le 13 novembre 2015.

DOI : 10.14428/emulations.cr.011

Distribution électronique : Université catholique de Louvain (Belgique) : ojs.uclouvain.be

(C) Cet article est mis à disposition selon les termes de la Licence Creative Commons Attribution, Pas d'Utilisation Commerciale 4.0 International. http://creativecommons.org/licenses/by-nc/4.0/

Éditeur : Émulations - Revue de sciences sociales / Presses universitaires de Louvain https://ojs.uclouvain.be/index.php/emulations

ISSN électronique : 1784-5734

UCL PRESSES

UNIVERSITAIRES

DE LOUVAIN 


\section{Juan José Bautista S. - ¿Qué significa pensar desde América Latina?: Hacia una racionalidad transmoderna y postcolonial}

Luis Martínez Andrade ${ }^{1}$

Recensé : Juan José Bautista S., ¿Qué significa pensar desde América Latina?: Hacia una racionalidad transmoderna y postcolonial, Madrid, Akal, 2014, 285 p.

Actuellement, l'essor de la pensée libérationniste et décoloniale (Bourguignon, Colin, Grosfoguel, 2014) suscite un grand intérêt de la part des intellectuels et des militants, qui témoigne, d'une part, de l'impasse de la théorie sociale eurocentrée et, d'autre part, de l'émergence d'une épistémologie du Sud. Or, la production théorique et épistémologique de la périphérie a toujours insisté sur le rôle de la raison critique, sur l'importance de la libération et la pertinence des projets alternatifs visant à tisser les liens sociaux et communautaires - liens brisés, depuis 1492, par cette modernité réellement existante. Les travaux de Juan José Bautista (2010 ; 2012), philosophe et sociologue bolivien, s'inscrivent bel et bien dans la continuité de cette pensée radicale. Ainsi, nous cherchons ici à mettre en lumières les idées principales qui se trouvent au cœur de son dernier ouvrage intitulé ¿Qué significa pensar desde América Latina?: Hacia una racionalidad transmoderna y postcolonial.

Composé de neuf chapitres, cet ouvrage approche l'Amérique latine à la fois comme un lieu d'énonciation «distinct» (p. 24) et comme un cas « extrême» (p. 67) de ce qui a été - et est encore aujourd'hui - la colonisation de la subjectivité des peuples. Le but de l'auteur est clair : il s'agit de produire des concepts et des catégories à même de contribuer à la mise en œuvre d'une « rationalité de la vie » qui s'opposerait à la rationalité raciste et dominatrice de la modernité. Bien entendu, cette rationalité de la vie n'est pas une création ex nihilo; elle est le fruit de l'accumulation, par les peuples opprimés, de savoirs, d'expériences et de traditions.

La première partie de l'ouvrage, intitulée « Del pensar », souligne principalement l’importance de l'Éthique de la libération qui a été développée par le philosophe Enrique Dussel (2002). Ce dernier, considéré comme le principal représentant de ce courant de

\footnotetext{
${ }^{1}$ Docteur en sociologie de l'École des hautes études en sciences sociales. Chercheur au CriDIS (Centre de Recherches interdisciplinaires Démocratie, Institutions, Subjectivité), Université catholique de Louvain.
} 
pensée par Carlos Beorlegui, est le meilleur exemple de la production d'un projet philosophique à portée universelle réalisée depuis l'Amérique latine (Bautista, 2014). Cependant, d'après Bautista, la pensée dusselienne a été la cible de critiques en raison de certains malentendus qu'il entend déconstruire. Il dénombre quatre catégories de critiques principales. La première réside dans l'assimilation que Dussel a faite de la pensée d'Emmanuel Levinas en tant que critique de l'ontologie de la modernité. Certes, la notion d'autrui chère à Levinas est cruciale dans l'architectonique discursive, c'est-à-dire dans le projet philosophique de Dussel, mais le fait de penser cet autrui comme l'extériorité radicalement niée (les populations indigènes et noires) par la modernité hégémonique a amené Dussel à proposer un autre cadre catégoriel (les notions d'analéctique, de corporalité ou de corporéité souffrante, le distinct, la trans-modernité, etc.). C'est en raison de cette assimilation de la pensée levinasienne que Dussel est considéré davantage comme un théologien que comme un philosophe (Bautista, 2014). La deuxième critique est liée à l'usage que Dussel fait de la catégorie du peuple. Or, c'est à partir d'une lecture approfondie de l'œuvre de Marx que Dussel distingue le «travail vivant» et le «travail objectivé». Ainsi, comme l'écrit Bautista, «Dussel peut affirmer que le "travail vivant" est le principe métaphysique réel et critique du capital - ce dernier étant pris en tant que totalité» (p. 32). La catégorie de peuple est donc comprise comme le pauper (pauvre). La troisième difficulté porte sur le dialogue entamé entre l'Éthique de la libération et l'Éthique du discours (Appel, Dussel, 2004). À cet égard, Bautista souligne le fait que Dussel prend au sérieux le «tournant pragmatique» proposé par Karl-Otto Appel (Saint-Georges, 2011), mais le fait à partir d'une perspective criticomatérielle des victimes. La quatrième critique fait référence à l'écriture de l'histoire. En effet, si, comme Walter Benjamin (1983 : 199) le suggère dans son texte Sur le concept d'histoire, «l'historiographe historiciste entre en empathie (Einfühlung) avec le vainqueur», un projet intellectuel d'émancipation devrait donc «brosser l'histoire à rebrousse-poil », c'est-à-dire pénétrer de plain-pied dans les débris et les ruines laissées par le train de la modernité afin de sauver le souvenir tel qu'il brille à l'instant d'un péril. En ce sens, Enrique Dussel propose de déconstruire (et de détruire) le récit historiographique hégémonique euro-centriste.

Dans la deuxième partie, qui a pour titre « De la crítica », J.J. Bautista présente les pensées d'Hugo Zemelman (et ses apports dans le domaine de l'épistémologie) et de Franz Hinkelammert. Selon nous, ce dernier a réalisé une critique impitoyable de la modernité-capitaliste - en tant que processus d'idolâtre. Né en 1931 en Allemagne, Franz Hinkelammert vit depuis 1963 en Amérique latine. Théologien et économiste, il a approfondi la théorie du fétichisme de Karl Marx afin d'articuler la Théorie critique (Horkheimer, Adorno et Benjamin) et la critique de la théorie (Hinkelammert, 1986; $1991 ; 1998$; 2002) pour saisir la dynamique meurtrière de la formation sociale hégémonique qu'est le capitalisme. 
De surcroît, c'est dans cette partie de l'ouvrage que figure, à nos yeux, le texte le plus intéressant. Le chapitre intitulé «Critique de l'idolâtrie de la modernité. Vers une théorie critique du fétichisme de la modernité » présente, à partir de la pensée de Franz Hinkelammert, les principales thèses de la théologie de la libération. Certes, différents travaux des théologiens et théologiennes de la libération ont retenu l'attention du monde académique dans les décennies 1970 et 1980, mais les perspectives ouvertes (telles que la théologie queer de Marcella Althaus-Reid, la théologie de la houe de Joseph Comblin, la théologie de la Terre proposée par Marcelo Barros et Ivo Poletto ou la théologie écologique prônée par Leonardo Boff) sont encore méconnues, du moins au sein du monde francophone. Ainsi, J. J. Bautista observe que la critique du capitalisme va de pair avec une critique de la rationalité moderne, car le noyau dur de cette modernité réellement existante est la dynamique sacrificielle des victimes (Hinkelammert, 1986). Il reconnait également l'importance de la critique du système légaliste faite par Hinkelammert. Comme nous le savons, la figure de Saint Paul a été réactualisée dans les débats contemporains, grâce aux travaux d'Alain Badiou, de Giorgio Agamben et de Slavoj Žižek. Cependant, l'exégèse de la pensée paulienne faite par Enrique Dussel (2011) ou par Franz Hinkelammert (2010) doit encore être étudiée plus en profondeur. Pour Hinkelammert, la critique paulienne de la Loi est un critique de la conception des «lois justes » car leur sens peut être détourné et, ainsi, les rendre injustes.

Finalement, «De la racionalidad», la dernière partie de l'ouvrage, livre quelques réflexions autour de la rationalité de la vie, de la diversité de temporalités, de la communauté trans-moderne, de l'intersubjectivité communautaire et de la notion du développement. L'auteur y invite à appréhender la nature comme sujet. Ainsi, Bautista nous propose de penser le passage de la société moderne vers une communauté trans-moderne, c'est-à-dire une formation sociale dont les diversités et les particularités ne sont pas chosifiées par la logique de la raison instrumentale. Pour ce faire, l'auteur mobilise la théorie du fétichisme - dont il se sert comme une théorie de la science critique - pour dénoncer la fausseté de la société moderne-capitaliste. Bautista juge que les autres temporalités non capitalistes sont d'une importance cruciale car elles nous permettent d'esquisser des formes de socialisation différentes. Face à la raison instrumentale moderne, il construit les bases de son projet théorique sur une rationalité de la vie. Autrement dit, il cherche à endiguer les mythes de cette modernité réellement existante, en s'appuyant pour ce faire sur les vaincus de l'histoire - au sens benjaminien du terme.

Certes, certains passages de cet ouvrage sont discutables. Par exemple, lorsque Bautista soutient que « le grand drame de la pensée critique du XXe siècle réside dans le fait que, même si elle était critique du capitalisme, elle est toujours restée à l'intérieur de la modernité ainsi que dans son cadre catégoriel, et elle a justifié la modernité et le modèle de société qu'elle abrite» (p. 226). Cette déclaration ne tient pas la route dès lors que l'on pense au projet intellectuel et politique de l'École de Francfort et, plus particulièrement, à l'œuvre de Walter Benjamin! Toutefois, cet ouvrage mérite d'être pris au 
sérieux par les chercheurs et chercheuses qui souhaitent s'opposer à la marchandisation de la connaissance et à la domination des savoirs néocoloniaux. Sorti il y a à peine un an, ce livre vient de remporter la dixième édition du Prix Libertador de la pensée critique. Créé en 2005 par l'ancien président vénézuélien Hugo Chávez, ce prix vise à encourager la pensée alternative et critique sur les situations de domination qui oppriment les peuples. Malgré sa parution récente, ¿Qué significa pensar desde América Latina? est déjà une pierre angulaire de la pensée critique latino-américaine contemporaine.

\section{Bibliographie}

BAUTISTA J. J. (2010) : Crítica de la Razón Boliviana: Elementos para una crítica de la subjetividad del boliviano con consciencia colonial, moderna y latinoamericana. La Paz : Rincón Ediciones.

BAUTISTA J. J. (2012) : Hacia la descolonización de la ciencia social Latinoamericana (cuatro ensayos metodológicos y epistemológicos). La Paz : Rincón Ediciones.

BENJAMIN W. (1983) : Essais (1935-1940). Paris : Denoël/Gonthier.

Bourguignon C., Colin P., Grosfoguel R. (2014) : Penser l'envers obscur de la modernité -Une anthologie de la pensée décoloniale latino-américaine. Limoges : Presses universitaires de Limoges et du Limousin.

DUSSEL E. (2002) : L'éthique de la libération à l'ère de la mondialisation et de l'exclusion. Paris : L’Harmattan.

DusSEL E. (2011) : Pablo de Tarso en la filosofía política actual y otros ensayos. México : Paulinas.

Hinkelammert F. (1986): The ideological weapons of death: A theological critique of capitalism. New York : Orbis.

HinKELAMMERT F. (1991) : Sacrificioshumanos y sociedad occidental. San José : DEI.

HinKelammert F. (1998) : El gritodelsujeto. San José : DEI.

HinkelammeRt F. (2002) : Critica de la razón utópica. Bilbao : Desclée de Brouwer.

HINKELAMMERT F., (2010) : La maldición que pesa sobre la ley. Las raíces del pensamiento crítico en Pablo de Tarso. San José : Arlekin.

SAint-Georges B., (2011): "Dussel et Appel: les enjeux d'un dialogue avorté». In Maesschalck M., Loute A. (dir.), Nouvelle critique sociale, Europe-Amérique Latine, Aller-Retour. Monza/Italie : Polimetrica, p. 65-89. 